\title{
Recovery of coupled networks after cascading failures
}

\author{
GAO Jiazi ${ }^{1}$, YIN Yongfeng ${ }^{1, *}$, FIONDELLA Lance ${ }^{2}$, and LIU Lijun ${ }^{1}$ \\ 1. School of Reliability and Systems Engineering, Beihang University, Beijing 100191, China; \\ 2. Department of Electrical \& Computer Engineering, University of Massachusetts, North Dartmouth MA 02747-2300, USA
}

\begin{abstract}
With society's increasing dependence on critical infrastructure such as power grids and communications systems, the robustness of these systems has attracted significant attention. Failure of some nodes can trigger a cascading failure, which completely fragments the network, necessitating recovery efforts to improve robustness of complex systems. Inspired by real-world scenarios, this paper proposes repair models after two kinds of network failures, namely complete and incomplete collapse. In both models, three kinds of repair strategies are possible, including random selection (RS), node selection based on single network node degree (SD), and node selection based on double network node degree (DD). We find that the node correlation in each of the two coupled networks affects repair efficiency. Numerical simulation and analysis results suggest that the repair node ratio and repair strategies may have a significant impact on the economics of the repair process. The results of this study thus provide insight into ways to improve the robustness of coupled networks after cascading failures.
\end{abstract}

Keywords: networks reliability, interdependent networks, recovery strategy, cascading failure.

DOI: $10.21629 /$ JSEE.2018.03.22

\section{Introduction}

Quality and reliability are two of the key factors affecting network success or failure [1,2]. Contemporary infrastructure systems such as power grids, the Internet, and transportation networks have become increasingly complex such that the failure of a few nodes may lead to paralysis of the entire network. Catastrophic effect such as the massive blackout of September 2003 that affected 58 million people in Italy [3] is but one such example.

Previous studies of the complex network theory focus on isolated networks such as the cascading model [3-5] as well as different control measures and defense strategies [6-9]. However, with the development of science and

Manuscript received December 21, 2016

*Corresponding author.

This work was supported by the National Natural Science Foundation of China (60972145), and the National Aerospace Science Foundation of China (20140751008). technology, various real world network infrastructures interact with each other. For example, power and communication networks are interdependent [10]. The robustness of coupled networks is quite different from that of isolated networks, where the state of one network depends on the state of the other and vice versa.

In 2010, Buldyrev et al. [11] studied the cascading failure of coupled networks, simulating the coupling failure phenomenon of the Italian power and communication systems. The model proposed assumed that when any node failed in one network, its corresponding coupled node in other networks also failed. In many cases, only nodes belonging to the giant component of both networks work normally after cascading failures. Thus, this research proposes the basic theoretical framework to study coupled networks.

Since then, much more efforts have focused on cascading failures of coupled networks. Parshani et al. [12] studied the coupling networks that the proportion of network A depends on network B as a networks coupling strength index, reducing the coupling strength to achieve improved robustness of coupled networks. Di et al. [13] found that the assortativity within a single network can decrease the robustness of the system. However, the robustness of a network can be improved by protecting the key node in the network. Cheng et al. [14] studied the nature of the coupling network and pointed out that different types of network coupling performance were even more vulnerable than the same type of network. Cao et al. [15] showed three link-addition strategies to enhance the robustness of cascading failures in coupled networks. Schneider et al. [16] proposed to choose the part of the autonomous nodes in the network to significantly improve the network robustness. They found that the network's ability to fight against cascading failures can be improved by selecting a number of autonomous nodes based on degree centrality and betweenness centrality. Tian et al. [17] found that assortative coupling in interconnected networks was more helpful to resist attacks. Furthermore, many impacts of cascading failures have been investigated, such as coupling patterns 
[18-21], control strategies [22-24], and load redistribution strategies $[25,26]$.

The studies discussed above, however, focused on the dependency network cascading failures model and its robustness. In the real world, effective measures will be taken to ensure network functions after various disturbances. Thus, it is necessary to determine how to repair the network efficiently. This paper seeks to answer this question with repair strategies and coupling patterns.

The remainder of this paper is organized as follows. In Section 2, we introduce a coupled network repair model based on different node selection strategies. In Section 3, we conduct numerical simulations of coupled networks and provide a detailed discussion. In Section 4, conclusions are provided.

\section{Recovery model and simulation}

\subsection{Recovery model in complete failure situations}

Based on the failure mechanism of coupled networks and node-based priority recovery strategies, Buldyrev et al. [11] proposed an interdependent network failure model which described the network cascading failures process. In this paper, we will take measures to restore the coupled networks and analyze the robustness of coupled

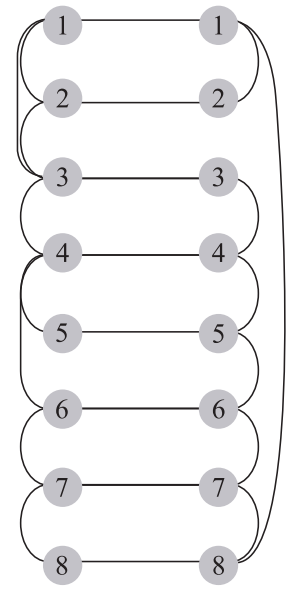

(a) Completely failed state

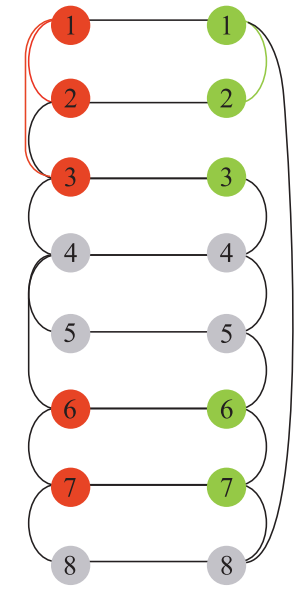

(b) Choosing nodes to restore networks under different restoration measures with different coupling patterns.

We extend the cascading model proposed by Gong et al. [27]. This paper discusses both completely failed coupled networks and partial failed coupled networks, comparing three different nodes selection strategies: random selection (RS), single node degree (SD) and double networks node degree (DD). In Fig. 1(a), we select a percentage $p_{r}$ nodes from either network which will be fully restored (the node is operational after repair). Meanwhile, the corresponding coupled node in the other network will be activated. Next, it must be determined whether the new cascading failure process will be generated in interdependent networks. The process is described as follows:

When the coupled networks fail completely, select a proportion $p_{r}$ of nodes to fully restore from network A and the counterpart nodes in network B will return to normal, and the dependency links between the networks will return to normal.

When all selected nodes are fully restored, due to the possible presence of a single small component, other situations may cause new cascading failures. The failure process continues until the network reaches a stable state, where the number of nodes surviving at this time $N_{S}$ (the number of survived nodes in a single network).

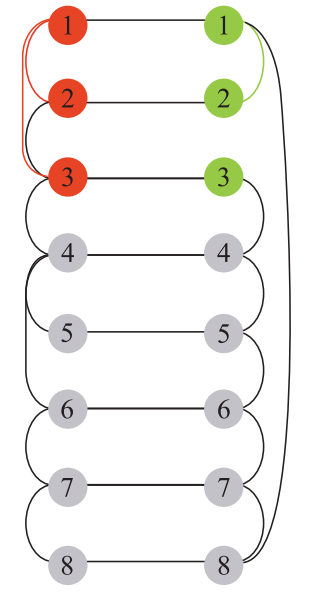

(c) Keeping the largest cluster

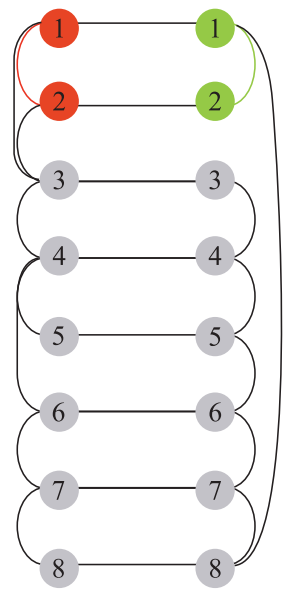

(d) Final coupled networks
Fig. 1 Recovery model in complete failure situations
As shown in Fig. 1, the left side of the network is network $A$ (shown in red), and the right side of the network is network B (shown in green). One-to-one correspondences between nodes of network $\mathrm{A}$ and $\mathrm{B}$, both networks have eight nodes. Fig. 1(a) is the completely failed state of the coupled networks, where all nodes are inactive; As shown in Fig. 1(b), choose nodes 1,2,3,6,7 at random to restore the network. The dependent links return to work, but the coupled networks do not belong to a connected cluster. Nodes
6 and 7 of the network A do not belong to the largest connected cluster [11], so nodes 6 and 7 remain failed. Node 3 in network B does not belong to 1, 2, or 6, 7 of connected clusters. The result of the final coupled networks after implementing the new cascading failure process reaches the steady state shown in Fig. 1(d).

\subsection{Recovery model in partial failure situations}

After the cascading failure, an incomplete state of collapse 
in coupled networks will be present, meaning there remains a certain percentage of active nodes in the networks. Thus, we should repair the failure nodes and analyze the influence of different node selection strategies.

As shown in Fig. 2(a), the nodes 4,5,6 of the coupled network are the survival nodes after cascading fail-

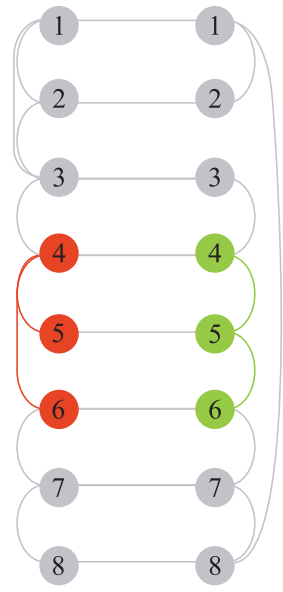

(a) Partial failure

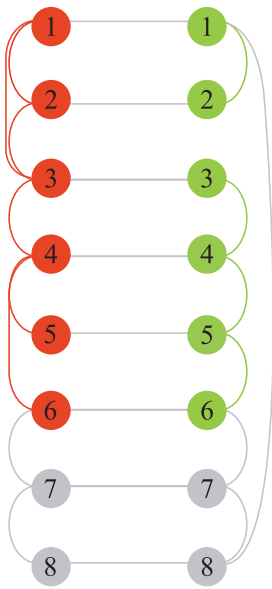

(b) Choosing nodes to restore
Fig. 2 Recovery model in partial failure situations a stable state.

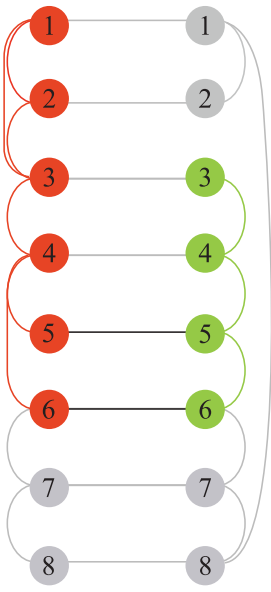

(c) Keeping the largest cluster

ures, then selected nodes 1,2,3 are fully restored; in Fig. 2(b), nodes 1 and 2 in network B do not belong to the largest connected cluster, according to the failure mechanism, these nodes fail, as shown in Fig. 2(c); the coupling nodes in network A fail. In Fig. 2(d), the network reaches

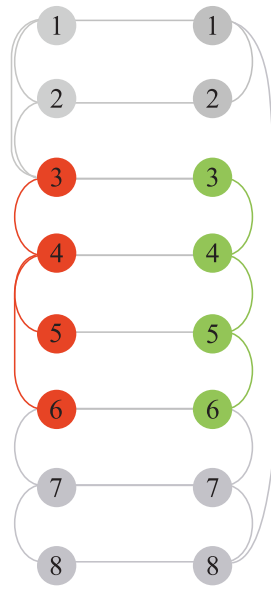

(d) Final coupled networks

\subsection{Three different recovery strategies}

Selected nodes may directly affect the survival rate of the network (number of nodes remaining work). Therefore, according to the relevant node attributes, we propose three kinds of node selection strategies: RS,SD and DD. All the strategies are explained as follows:

RS: Randomly select a proportion of $p_{r}$ nodes to fully restore in either network;

SD: Sort all nodes in the network in a descending order of degree. For example, to all the nodes in network A, the degree of $d_{1}^{A}, d_{2}^{A}, \ldots, d_{N}^{A}$ in a descending order, $d_{i}^{A}$ represents the degree of node $i$ in network $d_{1}^{A}$ and $N$ is the network size. According to the sorting result, we choose a small proportion of $p_{r}$ nodes to completely repair.

DD: Because the interdependent network model is fully coupled. It defines a one-to-one correspondence between nodes of networks A and B, as shown Fig. 1. This method adds the coupling nodes degree and then sorts, computed as follows:

$$
\left\{\begin{array}{l}
d_{1}=d_{1}^{A}+d_{1}^{B} \\
d_{2}=d_{2}^{A}+d_{2}^{B} \\
\vdots \\
d_{N}=d_{N}^{A}+d_{N}^{B}
\end{array} .\right.
$$

For $d_{1}, d_{2}, \ldots, d_{N}$ in a descending order of degree, select by a fraction $p_{r}$ of nodes to repair according to this order.

\subsection{Cascading process after recovery}

To compare the impact of the repair process after cascad- ing failures, the repair strategies are completely the same in the complete failure state and the incomplete failure state. However, we will select the inactive nodes to repair rather than the working nodes in networks.

According to [10], in the state of complete failures, cascading failures based on the node repair process can be described as

$$
\left\{\begin{array}{l}
\mu_{1}^{r}\left(p_{r}\right)=p_{r} \\
\mu_{n}^{r}\left(p_{r}\right)=q_{v, n}^{r} S_{A}^{r}\left(\mu_{n}^{r}\left(p_{r}\right)\right) p_{r} \\
\mu_{n}^{r}\left(p_{r}\right)=q_{\mu, n}^{r} S_{B}^{r}\left(\nu_{n-1}^{r}\left(p_{r}\right)\right) p_{r}
\end{array}\right.
$$

where $\nu_{n}^{r}\left(p_{r}\right) / \mu_{n}^{r}\left(p_{r}\right)$ denotes the proportion $p_{r}$ of nodes in network A to repair, the proportion of working nodes after $n-1$ iterations of network $\mathrm{A} / \mathrm{B}$, $S_{A}^{r}\left(\mu_{n}^{r}\left(p_{r}\right)\right) / S_{B}^{r}\left(\nu_{n-1}^{r}\left(p_{r}\right)\right)$ refers to the A/B network, the largest connected component. The remaining fraction of coupling nodes after repairing is $q_{v, n}^{r} / q_{\mu, n}^{r}$, when $\nu_{n}^{r}\left(p_{r}\right)=\nu_{n+1}^{r}\left(p_{r}\right), \mu_{n}^{r}\left(p_{r}\right)=\mu_{n+1}^{r}\left(p_{r}\right)$, and there is no further fragmentation in the repair process.

Under the condition of incomplete failures, where there exists a fraction of $p_{0}$ active nodes, the cascading failures in the repair process can be described as

$$
\left\{\begin{array}{l}
\mu_{1}^{r}\left(p_{r}+p_{0}\right)=p_{r}+p_{0} \\
\nu_{n}^{r}\left(p_{r}+p_{0}\right)=q_{v, n}^{r} S_{A}^{r}\left(\mu_{n}^{r}\left(p_{r}+p_{0}\right)\right)\left(p_{r}+p_{0}\right) \\
\mu_{n}^{r}\left(p_{r}+p_{0}\right)=q_{\mu, n}^{r} S_{B}^{r}\left(\nu_{n-1}^{r}\left(p_{r}+p_{0}\right)\right)\left(p_{r}+p_{0}\right)
\end{array} .\right.
$$

In order to analyze the failure process of the two states, as shown in Fig. 2, surviving nodes of the incomplete failure state will not fail during the repair process, while a 
restored node may fail again. Following this approach, the failure process can be simplified as follows:

$$
\left\{\begin{array}{l}
\varphi_{n}^{r}\left(p_{r}\right)=q_{\varphi, n}^{r} C_{A}^{r}\left(\psi_{n}^{r}\left(p_{r}\right)\right) p_{r} \\
\psi_{n}^{r}\left(p_{r}\right)=q_{\psi, n}^{r} C_{B}^{r}\left(\varphi_{n}^{r}\left(p_{r}\right)\right) p_{r}
\end{array}\right.
$$

where $\varphi_{n}^{r}\left(p_{r}\right) / \psi_{n}^{r}\left(p_{r}\right)$ and $q_{\varphi, n}^{r} / q_{\psi, n}^{r}$ are the same as described above and $C_{A}^{r}\left(\psi_{n}^{r}\left(p_{r}\right)\right) / C_{B}^{r}\left(\varphi_{n}^{r}\left(p_{r}\right)\right)$ defines the fraction of coupling nodes before the repair process. The limit must satisfy the equations $\varphi_{n}^{r}\left(p_{r}\right)=\psi_{n}^{r}\left(p_{r}\right)$ and $q_{\varphi, n}^{r}=q_{\psi, n}^{r}$, indicating that when there are no further cascading failures in the incomplete failure state, the network reaches a new steady state, where both the networks share the same node survival ratio: $\varphi_{n}^{r}\left(p_{r}\right)+p_{0}$.

To analyze the effect of different repair strategies, we propose the repair evaluation index: index of recovery (IoR), ratio of survival (RoS), which is defined as

$$
R o S=\frac{N_{s}}{N_{r}}
$$

where $N_{s}$ are the surviving nodes after the repair process, $N_{r}$ represents the number of nodes that we choose to repair, as shown in Fig. 2, $N_{s}=1, N_{r}=3$, and $R o S=\frac{1}{3}$.

\section{Simulation results and discussion}

In order to investigate the robustness of coupled networks after the repair process, the following examples are performed: Erdös-Rényi coupled-networks (ER-ER), scale free coupled-networks (SF-SF), and ER-SF coupled networks. The two networks are assumed to share the same size, $N_{A}=N_{B}=10000$ and the average degree, $K=4$, with coupling probability $p=1$. Each estimate is obtained by averaging over 50 realizations.

\subsection{Complete failure situation}

We randomly generate three modes of coupled networks and take different strategies to choose a fraction of nodes $p_{r}$ to repair. Ultimately, the network will reach a stable state after cascading failures.

First, we take four measures to select the nodes to be repaired: select nodes randomly, select nodes from network $\mathrm{A}$ in a descending order of degree, select nodes from network B in a descending order of degree, and select coupling nodes in a descending order of degree. As shown in Fig. 3, in the ER-ER coupled networks, random repair is least efficient. When the repair rate $p_{r}>0.58$, we can find the giant component, which promotes effective recovery. Repairing nodes in network A is the same as in network B, when $p_{r}>0.4$ and the network will recover from cascading failures more effectively than random selection. Next, we find that selecting coupling nodes in a descending order of degree is the most efficient way to repair the network in these four strategies, when $p_{r}>0.3$.

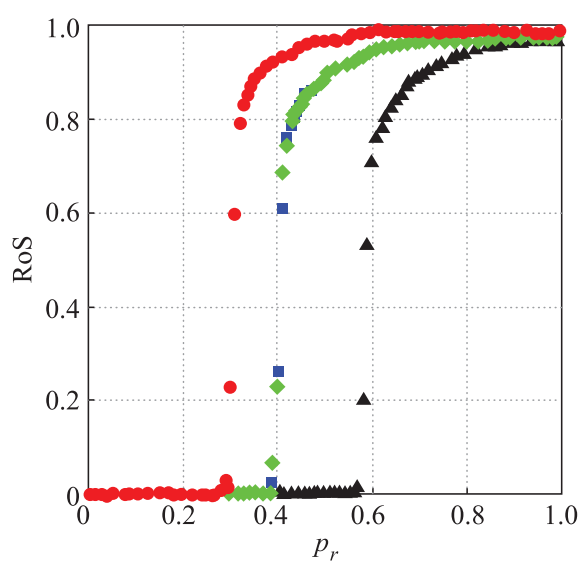

(a) Case of ER-ER networks

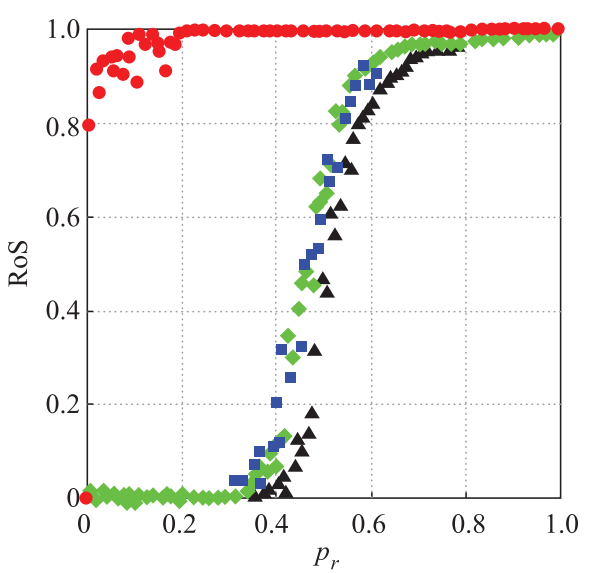

(b) Case of SF-SF networks

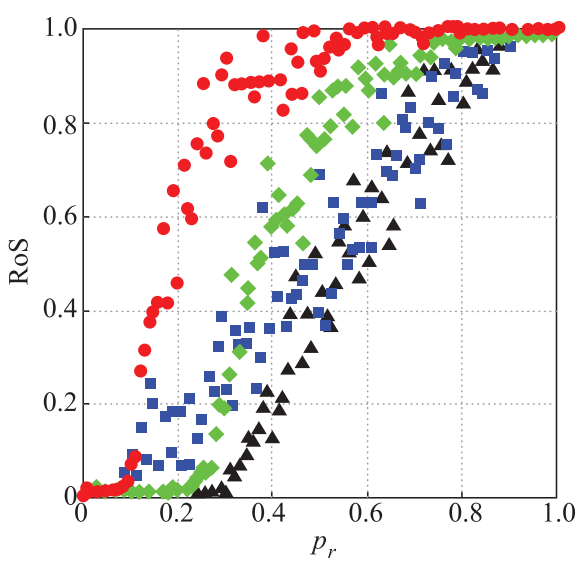

(c) Case of ER-SF networks

$\Delta: \mathrm{RS} ; \quad$ ॥: $\mathrm{SD}(\mathrm{A}) ; \quad$ : $\mathrm{SD}(\mathrm{B}) ; \quad \bullet: \mathrm{DD}$.

Fig. 3 Four strategies performed in complete failure state of three kinds of coupled networks

For SF-SF coupled networks, because the two single networks share the same characteristics, the coupling 
nodes degree repair strategy is the most efficient way to enhance the robustness of coupled networks. It is interesting to note that even when we choose a small fraction of nodes to repair, there still exist many active nodes. By analyzing the network model, we find that if the larger degree nodes in both networks are coupled together, this may promote restoration during the repair process.

While ER-SF coupled networks belong to the heterogeneous network, the experimental result fluctuates significantly. However, we can identify the trend in the curve. Random repair appears to be the worst choice. The single network degree repair strategy shows little improvement.

Fig. 3 shows four kinds of node selection strategies are performed in the complete failure state of three kinds of coupled networks. $p_{r}$ represents the fraction of nodes repaired. RoS represents the surviving nodes after repair measures. Red dots (RS) indicate randomly selected node repairs, blue squares ( $\mathrm{SD}(\mathrm{A})$ ) for nodes selected from network A, green diamonds ( $\mathrm{SD}(\mathrm{B})$ ) for nodes selected from network $\mathrm{B}$, and black triangles (DD) for nodes selected from coupling nodes in a descending order of degree.

Comparing the results of the three types of coupling patterns and repair strategies, we find there is a threshold $\theta$, when $p_{r}<\theta$, the repaired node will fail again, but when $p_{r} \geqslant \theta$, the repaired node may survive despite the cascading failure. For example, in ER-ER coupled networks, $\theta \approx$ 0.58 while in SF-SF coupled networks, $\theta \in(0.4,0.5)$, and in ER-SF coupled networks, $\theta \in(0.3,0.4)$. The threshold will be helpful to guide the recovery process of coupled networks.

\subsection{Partial failure situation}

Incomplete failure means that the coupled-networks do not collapse completely, but that some nodes remain in working status. We chose four different sizes of incompletely failed networks to analyze. First, we create the ER-ER, SF-SF, ER-SF and coupled-networks and then randomly remove $30 \%$ of nodes from the network. Next, the experiments are executed on the surviving nodes after cascading failures.

As shown in Fig. 4, Fig. 4(a), Fig. 4(b), Fig. 4(c) represent ER-ER, SF-SF and ER-SF coupled-networks, respectively. All networks remove $30 \%$ of nodes to create incomplete failure coupled-networks. Four kinds of repair strategies are simulated. $p_{r}$ represents the fraction of nodes repaired, RoS represents the survival nodes after repair measures. RS represents randomly repaired nodes, $\mathrm{SD}(\mathrm{A})$ selected from network A, SD(B) selected from network B, and DD selected from coupling nodes in a descending order of degree.

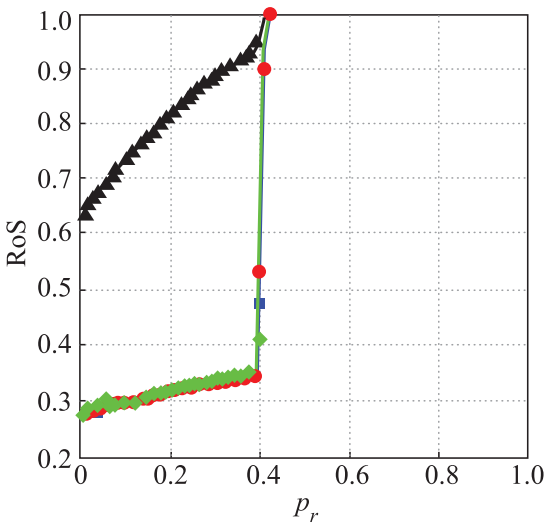

(a) Case of ER-ER networks

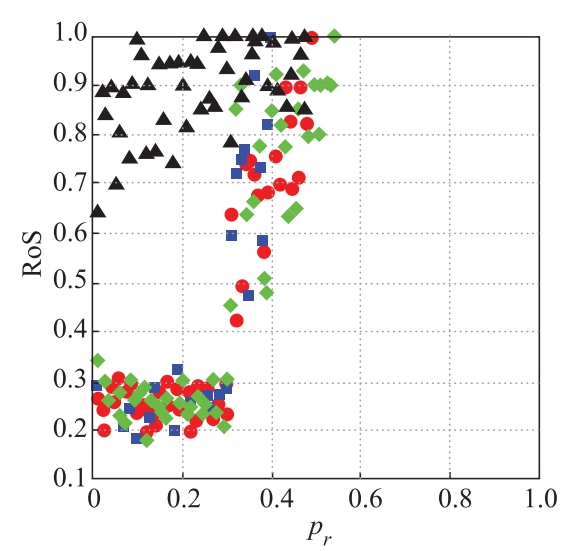

(b) Case of SF-SF networks

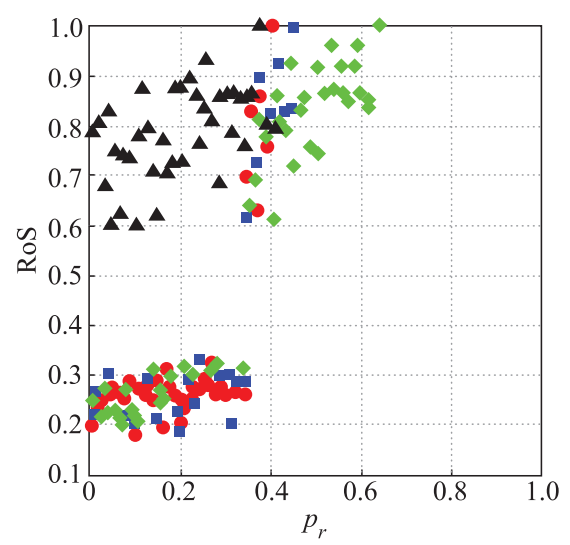

(c) Case of ER-SF networks $\Delta: \mathrm{RS} ; \quad$ : $\mathrm{SD}(\mathrm{A}) ; \quad \bullet \mathrm{SD}(\mathrm{B}) ; \quad \bullet: \mathrm{DD}$.

Fig. 4 Comparison of different strategies when $p_{f}=0.3$

Analyzing the result shown in Fig. 4, we find that there exists a threshold $\mu$ in the repair stategies. When $p_{r}<\mu$, the survival rate after repairing seems to be a fixed value, while for $p_{r} \geqslant \mu$, the survival rate exhibits a tendency of surge. For ER-ER coupled-networks, this threshold is $u=0.4$, when $p_{r}<0.4$ and the node survival rate is 0.3 . However, in SF-SF and ER-SF coupled networks, this threshold is $\mu \approx 0.3$, when $p_{r}>0.3$, the node survival rate 
is 0.25 . Unlike the complete failure conditions, the degree of coupling nodes does not achieve a better outcome, while the random selection strategy performs well. In three coupling patterns, the survival rate under different repair ratios are maintained above 0.6 . The reason may be the influence of the existing network prior to the repair process.

In order to study the influence of the initial network scale on the repair process, take ER-ER coupled-networks as an example. We remove $10 \%, 20 \%, 30 \%$, and $40 \%$ of nodes in the networks to generate the incomplete failure of couplednetworks, respectively and the networks are then repaired.

Fig. 5 shows four node selection strategies to repair networks, with different initial sizes. $p_{r}$ represents the fraction of nodes repaired. RoS represents the surviving nodes after repair measures. Fig. 5(a) is the random repair strategy for four initial sizes, Fig. 5(b) and Fig. 5(c) are single network repair strategies, Fig. 5(d) is the coupling nodes degree repair strategy. The fraction $p_{f}$ of nodes are removed from networks. Black triangles indicate $10 \%$, blue squares $20 \%$, green diamonds $30 \%$, and red circles $40 \%$.

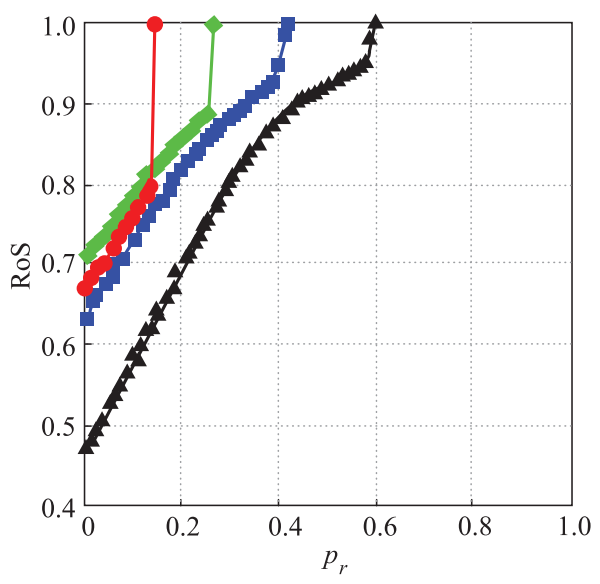

(a) RS strategy

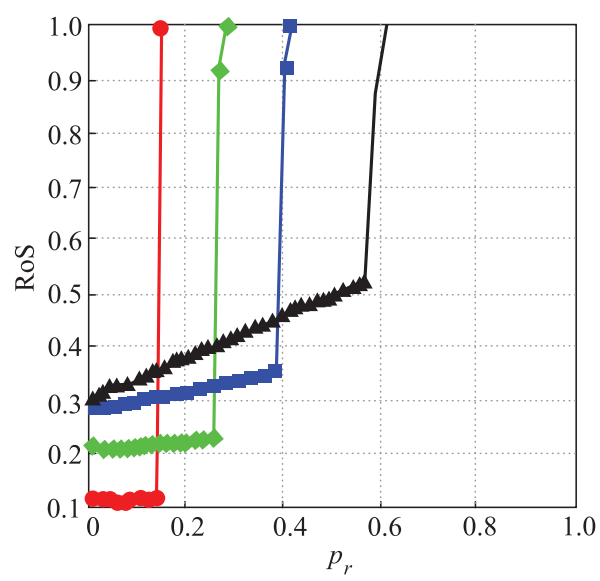

(b) $\mathrm{SD}(\mathrm{A})$ strategy

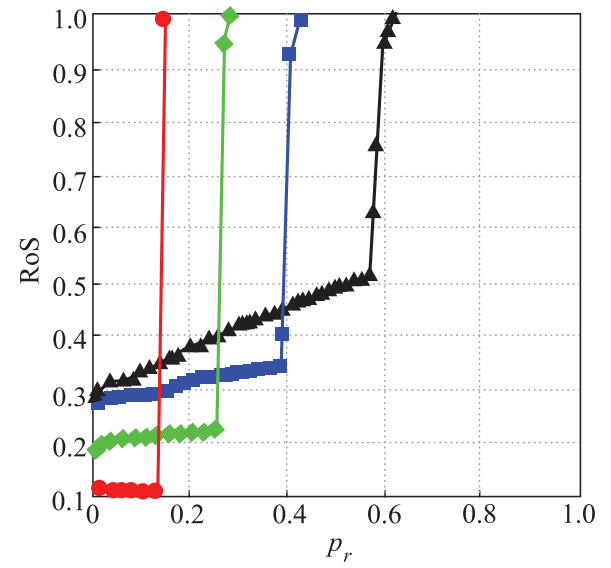

(c) $\mathrm{SD}(\mathrm{B})$ strategy

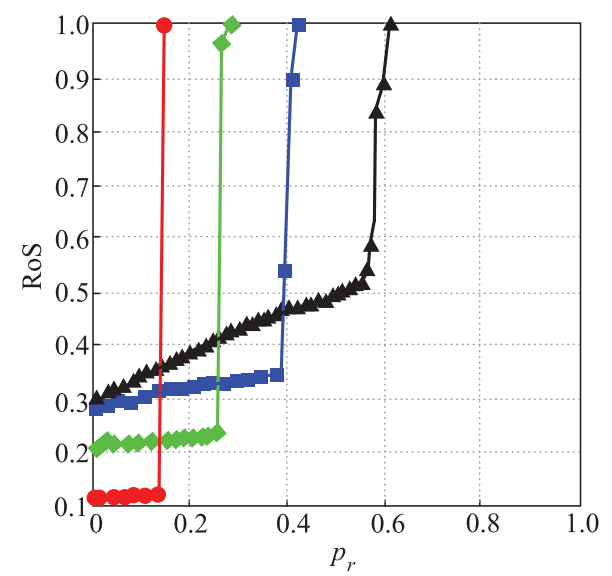

(d) DD strategy

$$
\longrightarrow: p_{f}: 0.1 ; \multimap: p_{f}: 0.3 ; \multimap: p_{f}: 0.2
$$

Fig. 5 Comparison different $p_{f}$ under the same strategy

As shown in Fig. 5, we find that the survival rate is independent on the network's initial size and the RoS is larger than 0.4. Comparing SD with DD, there exists a threshold, $\varepsilon \approx 1.5 p_{f}$, under the four network sizes considered. When $p_{r}<\varepsilon$, the survival rate RoS with repair rate $p_{r}$ increases linearly, whereas when $p_{r}=\varepsilon$ there exists a phase transition.

\section{Conclusions}

By applying the proposed repair strategies to coupled networks, we find that taking appropriate recovery measures could have a significant impact on the robustness of a network. In this paper, we have examined four repair strategies to investigate the restoration effect on completely failed coupled networks and incompletely failed networks. ER-ER, SF-SF and ER-SF network models are considered. The simulation of repair models suggests that the prioritizing node repaired by their coupling exhibits better results.

The following conclusions can be drawn from the model 
and numerical simulations:

Different network coupling patterns impact repair performance. Compared to the SF-SF and ER-SF models, the ER-ER model achieves better survival rates under the repair measures. The SF-SF model shows stronger robustness under the coupling nodes repair strategy.

The fraction of nodes repaired has a significant impact on the survival rate after recovery. In order to improve the efficiency of the repair process, we must take coupling patterns into consideration to determine whether it is worthwhile for maintenance and the proportion of the repair threshold.

Compared to the completely failed model, the survival rate of repaired nodes is severely affected by the existing nodes. Thus, we can take advantage of this characteristic to protect the key node with immunity to cascading failures, which will help to guide improvement of repair robustness of network systems.

\section{References}

[1] LIU L J, YIN Y F, LIU W, et al. Redundant design in interdependent networks. PLoS One, 2016, 11(10): e0164777.

[2] LI X D, YIN Y F, FIONDELLA L, et al. Software reliability assessment with correlated component failures under unified framework for software coupling measurement. Journal of Systems Engineering and Electronics, 2015, 26(5): 1114 1126.

[3] LATORA C P V, MARCHIORI M. A topological analysis of the Italian electric power grid. Physica A: Statistical Mechanics and its Applications, 2004, 338(1/2): 92-97.

[4] ALBERT R, ALBERT I, NAKARADO G L. Structural vulnerability of the North American power grid. Physical Review E, 2004, 69(2): 025103.

[5] RYAN K, ALBERT R. Modeling cascading failures in the North American power grid. The European Physical Journal B, 2005, 46(1): $101-107$.

[6] COHEN R, EREZ K, BENAVRAHAM D, et al. Resilience of the internet to random breakdowns. Physical Review Letters, 2000, 85(21): 4626-4628.

[7] MOTTER A E. Cascade control and defense in complex networks. Physical Review Letters, 2004, 93(9): 098701.

[8] WANG J. Mitigation of cascading failures on complex networks. Nonlinear Dynamics, 2012, 70(3): 1959-1967.

[9] LIU C, LI D, ZIO E. A modeling framework for system restoration from cascading failures. PLoS One, 2014, 9(12): e112363.

[10] RINALDI S M, PEERENBOOM J P, KELLY T K. Identifying, understanding, and analyzing critical infrastructure interdependencies. IEEE Control Systems, 2001, 21(6): 11-25.

[11] HAVLIN S, ARNÚJO N A M, BULDYREV S V, et al. Catastrophic cascade of failures in interdependent networks. Nature, 2010, 464(7291): $1025-1028$.

[12] PARSHANI R, BULDYREV S V, HAVLIN S. Interdependent networks: reducing the coupling strength leads to a change from a first to second order percolation transition. Physical Review Letters, 2010, 105(4): D48701.

[13] ZHOU D, STANLEY H E, D' AGOSTINO G, et al. Assortativity decreases the robustness of interdependent networks. Physical Review E, 2012, 86(2): 066103.

[14] CHENG Z, CAO J. Cascade of failures in interdependent networks coupled by different type networks. Physica A: Statisti- cal Mechanics and its Applications, 2015, 430: 193-200.

[15] CAO X B, HONG C, DU W B, et al. Improving the network robustness against cascading failures by adding links. Chaos, Solitons \& Fractals, 2013, 57(4): 35-40.

[16] SCHNEIDER C M, YAZDANI N, ARAUJO N A M, et al. Towards designing robust coupled networks. Scientific Reports, 2013, 3(24): $1-8$.

[17] TAN F, XIA Y X, WANG W P, et al. Cascading failures of loads in interconnected networks under intentional attack. Europhysics Letters, 2013, 102(2): 28009.

[18] SHAO J, BULDYREV S V, HAVLIN S, et al. Cascade of failures in coupled network systems with multiple supportdependence relations. Physical Review E, 2011, 83(3 Pt 2): 036116.

[19] TAN F, XIA Y, WEI Z. Robust-yet-fragile nature of interdependent networks. Physical Review E, 2015, 91(5): 052809.

[20] WANG J, LI Y, ZHENG Q. Cascading load model in interdependent networks with coupled strength. Physica A: Statistical Mechanics and its Applications, 2015, 430: 242-253.

[21] GAO J X, BULDYREV S V, HAVLIN S, et al. Robustness of a network formed by $n$ interdependent networks with a one-toone correspondence of dependent nodes. Physical Review E, 2012, 85(6): 066134.

[22] YANG Y, LI Z J, CHEN Y, et al. Improving the robustness of complex networks with preserving community structure. PLoS One, 2015, 10(2): e0116551.

[23] ZHANG W P, XIA Y X, LIU H Y, et al. Effect of network size on robustness of interconnected networks under targeted attack. Physica A: Statistical Mechanics and its Applications, 2015, 435: 80-88.

[24] STIPPINGER M, KERTÉSZ J. Enhancing resilience of interdependent networks by healing. Physica A: Statistical Mechanics and its Applications, 2014, 416: 481-487.

[25] QIU Y Z. Cascading dynamics with local weighted flow redistribution in interdependent networks. The European Physical Journal B, 2013, 86: 329.

[26] ZHAO Z, ZHANG P, YANG H. Cascading failures in interconnected networks with dynamical redistribution of loads. Physica A: Statistical Mechanics and its Applications, 2015, 433: $204-210$.

[27] GONG M G, MA M J, CAI Q, et al. Enhancing robustness of coupled networks under targeted recoveries. Scientific Reports, 2015, 5: 8439 .

\section{Biographies}

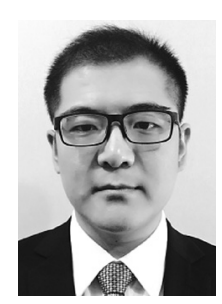

GAO Jiazi was born in 1984. He received his B.S. degree in communication engineering from North China Electric Power University in 2008. Now he is a postgraduate student in the School of Reliability and Systems Engineering, Beihang University. His current research interests include network reliability and software reliability engineering.

E-mail: 357209076@qq.com

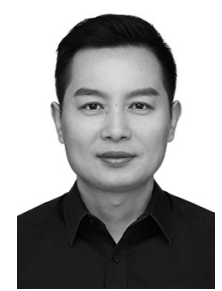

YIN Yongfeng was born in 1978. He received his M.S. and Ph.D. degrees in School of Reliability and Systems Enginneering, from Beihang University in 2003 and 2010 respectively. He went to Colorado State University as a visiting scholar from 2015 to 2016. Now he is an associate professor of Beihang University. His research interests include systems engineering, software testing and verification techniques, and software reliability engineering.

E-mail: yyf@buaa.edu.cn 


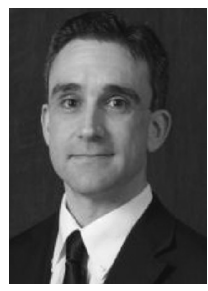

FIONDELLA Lance was born in 1975. He received his M.S. and Ph.D. degrees in computer science and engineering, from the University of Connecticut, Storrs, CT, USA, in 2003 and 2012, respectively. Presently, he is an assistant professor at the University of Massachusetts Dartmouth. His research interests include reliability and transportation engineering. He has published over 50 peerreviewed journal and conference papers. Four of his conference papers have been recognized with awards. He is a member of the IEEE Reliability Society and serves as the vice-chair of Standard 1633, Recommended Practice on Software Reliability.

E-mail: 1fiondella@umassd.edu

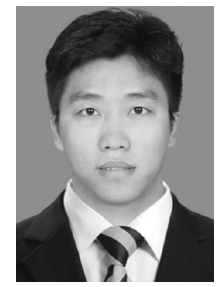

LIU Lijun was born in 1991. He received his B.S. degree in reliability engineering from Harbin Engineering University in 2014. Now he is a postgraduate student in the School of Reliability and Systems Engineering, Beihang University. His current research interests include network reliability and software reliability engineering.

E-mail: 164497197@qq.com 